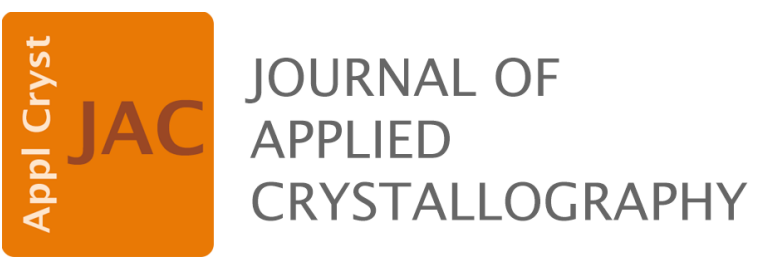

Volume 49 (2016)

Supporting information for article:

Accuracy in Rietveld quantitative phase analysis: A comparative study of Mo and Cu strictly monochromatic radiations

L. León-Reina, M. García-Maté, G. Álvarez-Pinazo, I. Santacruz, O. Vallcorba, A. G. De la Torre and M. A. G. Aranda 


\section{Accuracy in Rietveld quantitative phase analysis. A comparative study of Mo and Cu strictly monochromatic radiations}

L. León-Reina ${ }^{1}$, M. García-Matée ${ }^{2,3}$, G. Álvarez-Pinazo ${ }^{2,3}$, I. Santacruz ${ }^{2}$, O. Vallcorba ${ }^{4}$, A. G. De la Torre ${ }^{2}$, M. A. G. Aranda ${ }^{2,4 *}$

\footnotetext{
${ }^{1}$ Servicios Centrales de Apoyo a la Investigación, Universidad de Málaga, 29071-Málaga, Spain.

${ }^{2}$ Departamento de Química Inorgánica, Cristalografía y Mineralogía, Universidad de Málaga, 29071Málaga, Spain.

${ }^{3}$ X-Ray Data Services S.L., Edificio GREEN RAY, $1^{\text {a }}$ planta, Avda. Louis Pasteur, 47 (Ampliación Campus Teatinos), 29010-Málaga, Spain.

${ }^{4}$ ALBA synchrotron, Carrer de la Lum, 2-26, E-08290 Cerdanyola, Barcelona, Spain.
}

This supporting information contains:

Table S1. Refined profile parameters and refined March-Dollase preferred orientation parameter for Mo-K $\alpha_{1}$ patterns of organic phases (Glucose: G; Fructose: F; Lactose: L; Xylose: X).

Table S2. Refined profile parameters and refined March-Dollase preferred orientation parameter for Mo-K $\alpha_{1}$ pattern of inorganic single phases (Calcite: C; Quartz: Q; Insoluble anhydrite: i-A; ZnO).

Table S3. Rietveld quantitative phase analysis, refined profile parameters and refined March-Dollase preferred orientation parameter for Mo-K $\alpha_{1}$ pattern of gypsum sample.

Table S4. Rietveld quantitative phase analysis of CGpQ 0.12A mixture obtained from three independent $\mathrm{Mo}-\mathrm{K} \alpha_{1}$ and $\mathrm{Cu}-\mathrm{K} \alpha_{1}$ patterns. Numbers between brackets are the mathematical errors given by Rietveld program calculations.

Table S5. Rietveld quantitative phase analysis of CGPQ_0.12A mixture obtained from SXRPD patterns collected in three different positions in the capillary sample holder. Numbers between brackets are the mathematical errors given by Rietveld calculations. The mean value and the standard deviation of the three analyses are also given.

Table S6. Rietveld quantitative phase analysis of GFL_0.12X mixture obtained from three independent $\mathrm{Mo}-\mathrm{K} \alpha_{1}$ and $\mathrm{Cu}-\mathrm{K} \alpha_{1}$ patterns. Numbers between brackets are the mathematical errors given by Rietveld calculations. 
Table S7. Rietveld quantitative phase analysis of GFL_0.12X mixture obtained from SXRPD patterns collected in three different positions in the capillary sample holder. Numbers between brackets are the mathematical errors given by Rietveld calculations. The mean value and the standard deviation of the three analyses are also given.

Figure S1. Figure S1. Mo-radiation Rietveld plot for glucose.

Figure S2. Mo-K $\alpha_{1}$ radiation Rietveld plot for fructose.

Figure S3. Mo-K $\alpha_{1}$ radiation Rietveld plot for lactose.

Figure S4. Mo-K $\alpha_{1}$ radiation Rietveld plot for xylose.

Figure S5. Mo-K $\alpha_{1}$ radiation Rietveld plot for quartz.

Figure S6. Mo-K $\alpha_{1}$ radiation Rietveld plot for calcite.

Figure S7. Mo-K $\alpha_{1}$ radiation Rietveld plot for insoluble anhydrite.

Figure S8. Mo-K $\alpha_{1}$ radiation Rietveld plot for zincite.

Figure S9. Scanning electron microscopy micrographs for (a) micronized gypsum, and (b) ground single crystal gypsum. (c) Mo-K $\alpha_{1}$ radiation Rietveld plot for micronized gypsum.

Figure S10. Mo-K $\alpha_{1}$ raw pattern (black) for glucose collected in transmission geometry. Cu-K $\alpha_{1}$ raw pattern (blue) for glucose collected in reflection geometry. 
Table S1. Refined profile parameters and refined March-Dollase preferred orientation parameter for Mo-K $\alpha_{1}$ patterns of organic phases (Glucose: G; Fructose: F; Lactose: L; Xylose: X).

\begin{tabular}{ccccccccc}
\hline Phase & $\begin{array}{c}\mathbf{G U} \\
\left.\mathbf{( 0 . 0 1}^{\mathbf{0}}\right)^{2}\end{array}$ & $\begin{array}{c}\mathbf{G V} \\
/\left(\mathbf{0 . 0 1}^{\mathbf{0}}\right)^{2}\end{array}$ & $\begin{array}{c}\mathbf{G W} \\
/\left(\mathbf{0 . 0 1}^{\mathbf{0}}\right)^{2}\end{array}$ & $\begin{array}{c}\mathbf{L Y} \\
/\left(\mathbf{0 . 0 1}^{\mathbf{0}}\right)\end{array}$ & $\mathbf{S} / \mathbf{L}$ & $\mathbf{H} / \mathbf{L}$ & $\begin{array}{c}\text { STEC } \\
\left./ \mathbf{( 0 . 0 1}^{\mathbf{}}\right)\end{array}$ & PO \\
\hline $\mathbf{G}$ & $201(13)$ & $-16(3)$ & $2.7(2)$ & $6.7(2)$ & 0.015 & 0.014 & - & $1.040(1)^{*}$ \\
$\mathbf{F}$ & $159(3)$ & - & $1.93(3)$ & $0.8(2)$ & 0.014 & 0.015 & - & - \\
$\mathbf{L}$ & $321(17)$ & $-33(4)$ & $3.3(2)$ & $6.0(4)$ & 0.014 & 0.014 & $10.6(5)^{\#}$ & $1.120(1)^{*}$ \\
$\mathbf{X}$ & $377(20)$ & $-36(5)$ & $3.5(2)$ & $3.2(3)$ & 0.015 & 0.014 & - & -
\end{tabular}

* March-Dollase coefficient along [001] axis. ${ }^{\#}$ Anisotropic peak broadening direction for ellipsoidal correction [001].

Table S2. Refined profile parameters and refined March-Dollase preferred orientation parameter for Mo-K $\alpha_{1}$ pattern of inorganic single phases (Calcite: C; Quartz: Q; Insoluble anhydrite: i-A; ZnO).

\begin{tabular}{|c|c|c|c|c|c|c|c|c|}
\hline Phase & $\begin{array}{c}\text { GU } \\
\left(\mathbf{0 . 0 1}^{\mathbf{l}}\right)^{2} \\
\end{array}$ & $\begin{array}{c}\text { GV } \\
\left(0^{0.01}\right)^{2}\end{array}$ & $\begin{array}{c}\text { GW } \\
\left(0^{0.01^{\circ}}\right)^{2}\end{array}$ & $\begin{array}{c}\mathbf{L Y} \\
\left.(\mathbf{0 . 0 1})^{\circ}\right)\end{array}$ & $\mathrm{S} / \mathrm{L}$ & $\mathbf{H} / \mathbf{L}$ & $\begin{array}{l}\text { STEC } \\
\left(\mathbf{0 . 0 1}^{\circ}\right)\end{array}$ & PO \\
\hline $\mathbf{C}$ & 10(5) & $-9(2)$ & $2.1(2)$ & $2.7(2)$ & 0.015 & 0.015 & $-1.6(3)^{\#}$ & $1.050(7)^{*}$ \\
\hline $\mathbf{Q}$ & 95(5) & $-25(2)$ & $3.2(1)$ & $12.0(2)$ & 0.014 & 0.014 & $\&$ & $1.120(1)^{*}$ \\
\hline i-A@ & $106(2)$ & - & $1.30(4)$ & $2.3(2)$ & 0.015 & 0.015 & $8.7(3)^{\varsigma}$ & $1.040(2)^{\$}$ \\
\hline $\mathrm{ZnO}$ & 32(1) & - & 2.03(6) & $9.5(2)$ & 0.015 & 0.015 & $1.73(6)^{\epsilon}$ & $1.040(2)^{\$}$ \\
\hline
\end{tabular}

*March-Dollase coefficient along [104] axis. ${ }^{\$}$ March-Dollase coefficient along [010] axis. \#Anisotropic peak broadening direction for ellipsoidal correction [010]. \&Anisotropic peak broadening direction for ellipsoidal correction [101] using PTEC $\left(0.01^{\circ}\right)$ parameter: $0.83(7)$. ${ }^{\varsigma}$ Anisotropic peak broadening direction for ellipsoidal correction [100]. ${ }^{€}$ Anisotropic peak broadening direction for ellipsoidal correction [100] jointly with PTEC $\left(0.01^{\circ}\right)$ parameter: 6.4(4). @ It also contains $0.32(3) \mathrm{wt} \%$ of $\mathrm{SiO}_{2}, 0.86(3) \mathrm{wt} \%$ of $\mathrm{Cu}_{5} \mathrm{FeS}_{4}$ (ICSD code \# 1963) and $0.33(5) \mathrm{wt} \%$ of $\mathrm{SrSO}_{4}$. 
Table S3. Rietveld quantitative phase analysis, refined profile parameters and refined MarchDollase preferred orientation parameter for Mo-K $\alpha_{1}$ pattern of micronized gypsum sample.

\begin{tabular}{|c|c|c|c|c|c|c|c|c|c|}
\hline Phase & Wt\% & $\begin{array}{c}\text { GU } \\
\left(0^{0.01^{0}}\right)^{2}\end{array}$ & $\begin{array}{c}\text { GV } \\
\left(0.01^{\circ}\right)^{2}\end{array}$ & $\begin{array}{c}\text { GW } \\
\left(0^{0.01}\right)^{2} \\
\end{array}$ & $\begin{array}{c}\mathbf{L Y} \\
\left.(\mathbf{0 . 0 1})^{\circ}\right)\end{array}$ & $\mathbf{S} / \mathbf{L}$ & $H / L$ & $\begin{array}{l}\text { STEC } \\
\left(\mathbf{( 0 . 0 1}^{\circ}\right) \\
\end{array}$ & PO \\
\hline Gp & 93.83(2) & 129(6) & $-13(2)$ & $2.5(1)$ & $6.9(2)$ & 0.014 & 0.015 & - & $1.211(2)^{\$}$ \\
\hline s-A & $2.25(4)$ & 118(-) & $-10(-)$ & $2.2(-)$ & $7.8(-)$ & 0.014 & 0.015 & - & - \\
\hline $\mathrm{SrSO}_{4}$ & $1.13(4)$ & $133(-)$ & $-27(-)$ & $3.3(-)$ & $15.5(-)$ & 0.014 & 0.015 & - & - \\
\hline $\mathbf{Q}$ & $0.94(3)$ & $85(-)$ & $-23(-)$ & $3.0(-)$ & $13.0(-)$ & 0.014 & 0.015 & - & - \\
\hline C & $0.65(4)$ & $85(-)$ & $-23(-)$ & $3.0(-)$ & $13.0(-)$ & 0.014 & 0.015 & - & - \\
\hline i-A & $0.65(3)$ & $85(-)$ & $-23(-)$ & $3.0(-)$ & $13.0(-)$ & 0.014 & 0.015 & - & - \\
\hline $\mathrm{Na}_{2} \mathrm{SO}_{4}$ & $0.54(6)$ & $100(-)$ & $-26(-)$ & $3.0(-)$ & $15.0(-)$ & 0.014 & 0.015 & - & - \\
\hline
\end{tabular}

${ }^{\$}$ March-Dollase coefficient along [010] axis.

Table S4. Rietveld quantitative phase analysis of CGpQ_0.12A mixture obtained from three independent Mo-K $\alpha_{1}$ and $\mathrm{Cu}-\mathrm{K} \alpha_{1}$ patterns. Numbers between brackets are the mathematical errors given by Rietveld program calculations. Absolute values of the Kullback-Liebler (AKLD) for each mixture and the KLD value for i-anhydrite are also included.

\begin{tabular}{|c|c|c|c|c|c|c|c|}
\hline \multirow[b]{2}{*}{ Phase } & \multirow[b]{2}{*}{ Wt\% } & \multicolumn{3}{|c|}{ Mo-LXRPD } & \multicolumn{3}{|c|}{ Cu-LXRPD } \\
\hline & & $\# 1$ & $\# 2$ & \#3 & $\# 1$ & \#2 & \#3 \\
\hline C & 32.86 & 33.6(1) & 33.6(1) & $33.5(1)$ & 29.9(1) & $29.9(1)$ & $30.1(1)$ \\
\hline Gp & 31.67 & $30.4(1)$ & $30.3(1)$ & $30.4(1)$ & 34.1(1) & $34.0(1)$ & 33.5(1) \\
\hline $\mathbf{Q}$ & 34.17 & $34.8(1)$ & $34.9(1)$ & $34.8(1)$ & 34.3(1) & $34.4(1)$ & $34.7(1)$ \\
\hline s-A & 0.79 & $0.55(3)$ & $0.58(3)$ & $0.59(2)$ & $0.80(5)$ & $0.77(4)$ & $0.76(5)$ \\
\hline $\mathrm{SrSO}_{4}$ & 0.39 & $0.39(4)$ & $0.41(4)$ & $0.41(1)$ & $0.67(5)$ & $0.64(5)$ & $0.59(5)$ \\
\hline i-A & 0.12 & $0.28(3)$ & $0.26(2)$ & $0.29(2)$ & $0.22(3)$ & $0.25(3)$ & $0.26(3)$ \\
\hline AKLDsum & & 0.0304 & 0.0321 & 0.0291 & 0.0586 & 0.0588 & 0.0547 \\
\hline (i-A) KLD & & -0.0010 & -0.0009 & -0.0011 & -0.0007 & -0.0009 & -0.0009 \\
\hline
\end{tabular}


Table S5. Rietveld quantitative phase analysis of CGpQ_0.12A mixture obtained from SXRPD patterns collected in three different positions in the capillary sample holder. Numbers between brackets are the mathematical errors given by Rietveld calculations. The mean value and the standard deviation of the three analyses are also given. Absolute values of the Kullback-Liebler (AKLD) for each mixture and the KLD value for i-anhydrite are also included.

\begin{tabular}{ccccccc}
\hline Phase & Wt $\%$ & POS1 & POS2 & POS3 & Mean value & Standard deviation \\
\hline C & $\mathbf{3 2 . 8 6}$ & $33.90(5)$ & $34.14(6)$ & $33.97(5)$ & 34.0 & 0.1 \\
Gp & $\mathbf{3 1 . 6 7}$ & $31.24(7)$ & $30.98(7)$ & $31.85(7)$ & 31.4 & 0.4 \\
$\mathbf{Q}$ & $\mathbf{3 4 . 1 7}$ & $33.82(5)$ & $33.80(6)$ & $33.14(5)$ & 33.6 & 0.4 \\
s-A & $\mathbf{0 . 7 9}$ & $0.64(2)$ & $0.70(2)$ & $0.65(2)$ & 0.7 & 0.03 \\
SrSO & $\mathbf{0 . 3 9}$ & $0.20(1)$ & $0.19(1)$ & $0.20(1)$ & 0.2 & 0.006 \\
i-A & $\mathbf{0 . 1 2}$ & $0.21(2)$ & $0.19(2)$ & $0.19(1)$ & 0.2 & 0.01 \\
AKLDsum & & 0.0210 & 0.0275 & 0.0242 & & \\
(i-A) KLD & & 0.0006 & 0.0005 & 0.0005 & & \\
\hline
\end{tabular}


Table S6. Rietveld quantitative phase analysis of GFL_0.12X mixture obtained from three independent Mo-K $\alpha_{1}$ and $\mathrm{Cu}-\mathrm{K} \alpha_{1}$ patterns. Numbers between brackets are the mathematical errors given by Rietveld calculations. Absolute values of the Kullback-Liebler (AKLD) for each mixture and the KLD value for xylose are also included.

\begin{tabular}{|c|c|c|c|c|c|c|c|}
\hline \multirow[b]{2}{*}{ Phase } & \multirow[b]{2}{*}{ Wt\% } & \multicolumn{3}{|c|}{ Mo-LXRPD } & \multicolumn{3}{|c|}{ Cu-LXRPD } \\
\hline & & $\# 1$ & \#2 & $\# 3$ & $\# 1$ & \#2 & $\# 3$ \\
\hline G & 33.36 & $32.3(2)$ & $33.5(2)$ & $32.4(2)$ & $33.3(3)$ & $31.5(3)$ & $32.8(2)$ \\
\hline $\mathbf{F}$ & 33.46 & $32.7(1)$ & 33.1(1) & 33.1(1) & $34.0(2)$ & $33.9(3)$ & $33.2(2)$ \\
\hline $\mathbf{L}$ & 33.06 & $34.8(2)$ & $33.1(2)$ & $34.5(2)$ & $32.4(2)$ & $34.3(3)$ & $33.6(2)$ \\
\hline $\mathbf{X}$ & 0.12 & $0.17(5)$ & $0.27(5)$ & $0.11(5)$ & $0.35(9)$ & $0.28(10)$ & $0.40(8)$ \\
\hline AKLDsum & & 0.0358 & 0.0064 & 0.0276 & 0.0139 & 0.0367 & 0.0151 \\
\hline (X) KLD & & -0.0004 & -0.0010 & 0.0001 & -0.0013 & -0.0010 & -0.0014 \\
\hline
\end{tabular}


Table S7. Rietveld quantitative phase analysis of GFL_0.12X mixture obtained from SXRPD patterns collected in three different positions in the capillary sample holder. Numbers between brackets are the mathematical errors given by Rietveld calculations. The mean value and the standard deviation of the three analyses are also given.

\begin{tabular}{cccccc}
\hline Phase & POS1 & POS2 & POS3 & Mean value & Standard deviation \\
\hline G & $33.0(2)$ & $39.7(2)$ & $32.6(1)$ & 35.1 & 4.0 \\
F & $28.4(1)$ & $28.4(1)$ & $32.2(1)$ & 29.7 & 2.3 \\
L & $38.6(2)$ & $31.9(2)$ & $35.2(2)$ & 35.2 & 3.4 \\
X & - & - & - & - & - \\
\hline
\end{tabular}




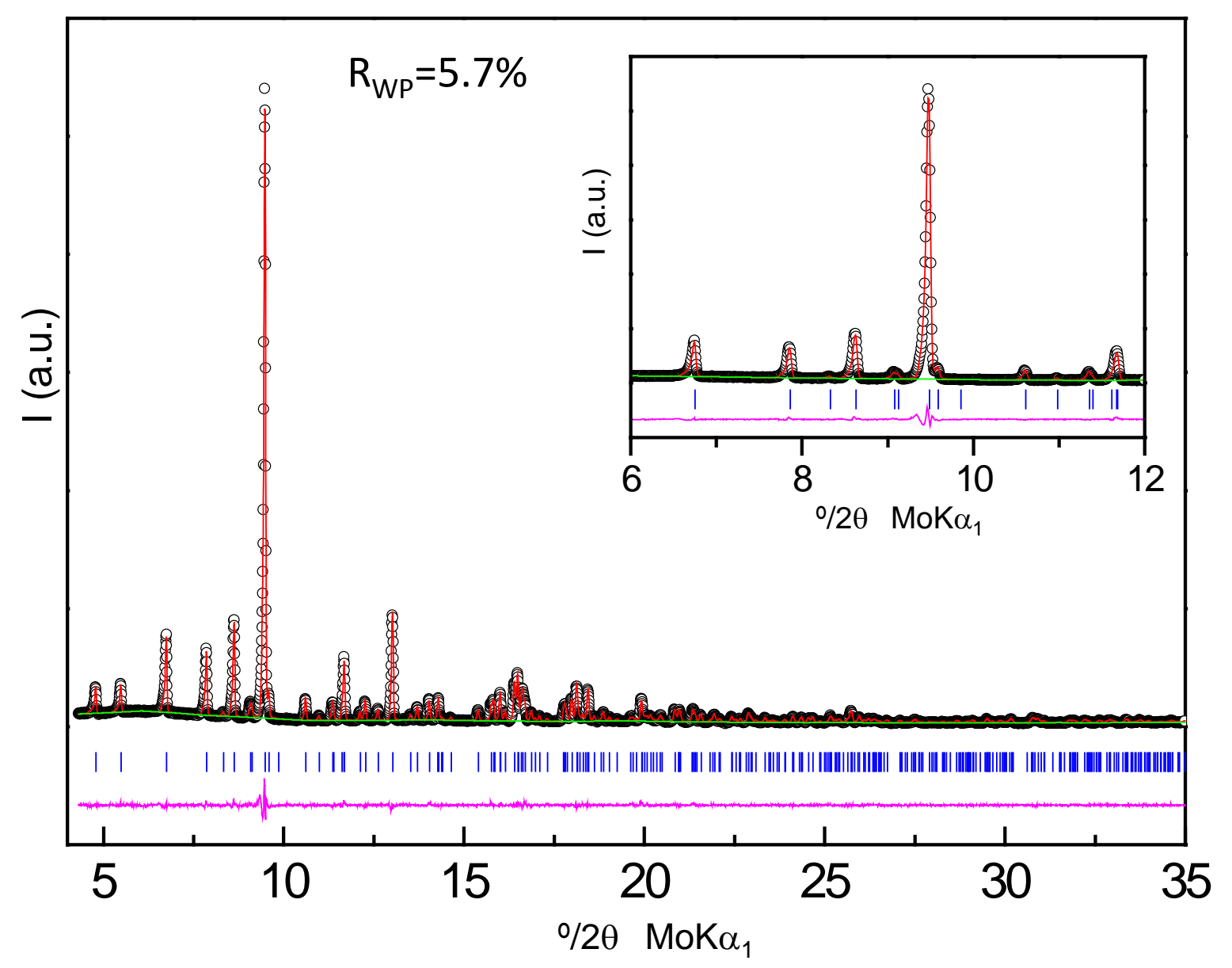

Figure S1. Mo-K $\alpha_{1}$ radiation Rietveld plot for glucose. 


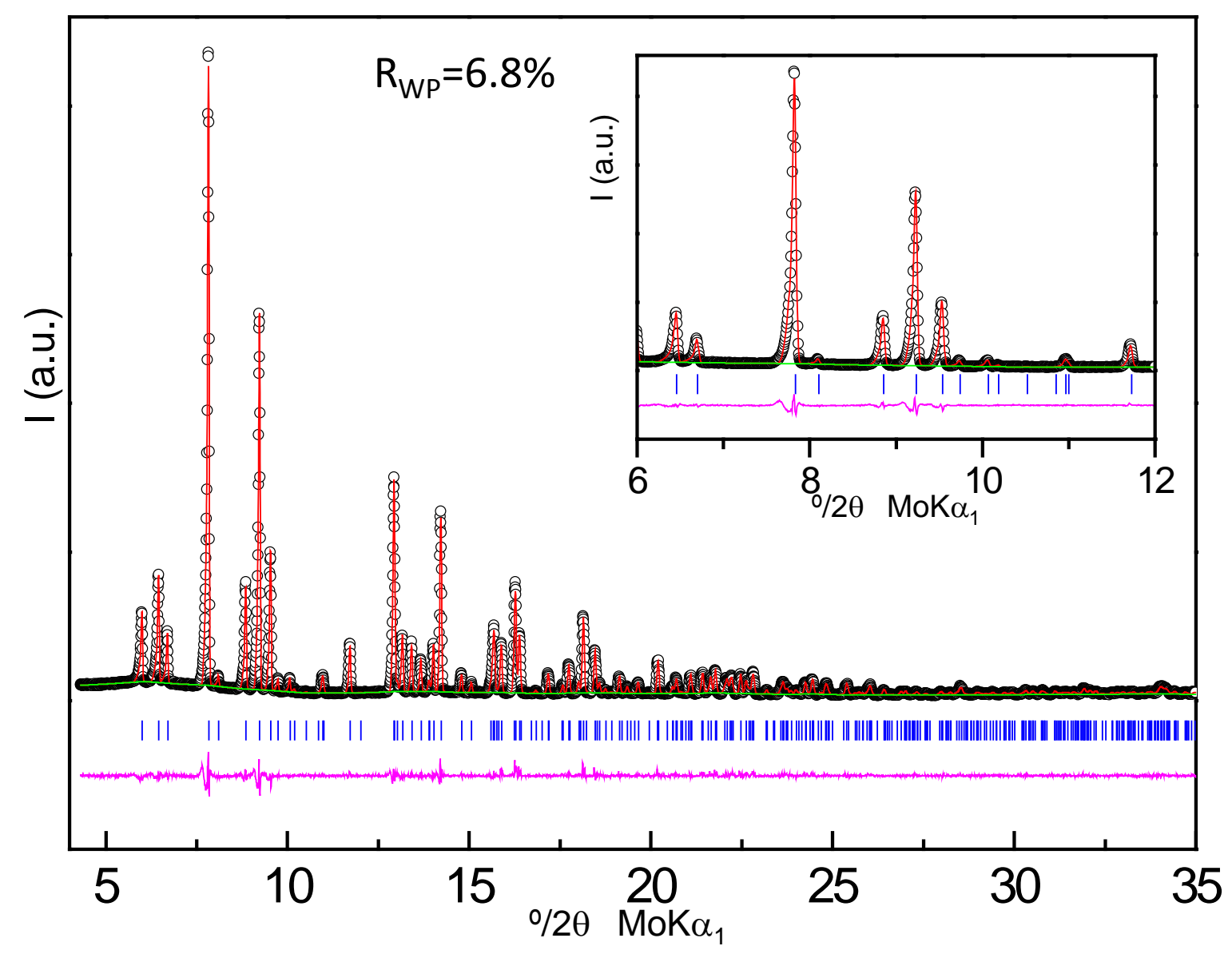

Figure S2. Mo-K $\alpha_{1}$ radiation Rietveld plot for fructose. 


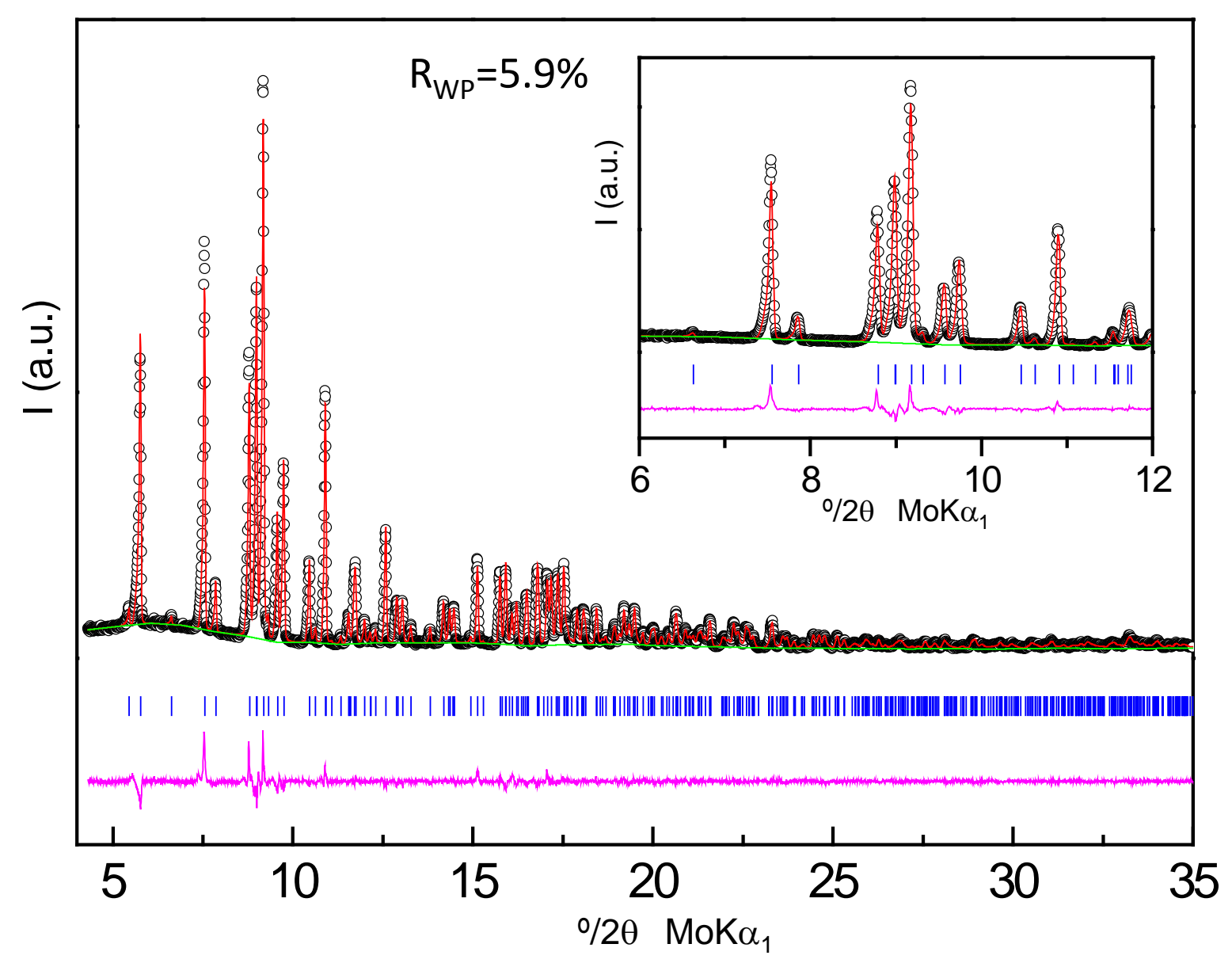

Figure S3. Mo- $\mathrm{K} \alpha_{1}$ radiation Rietveld plot for lactose. 


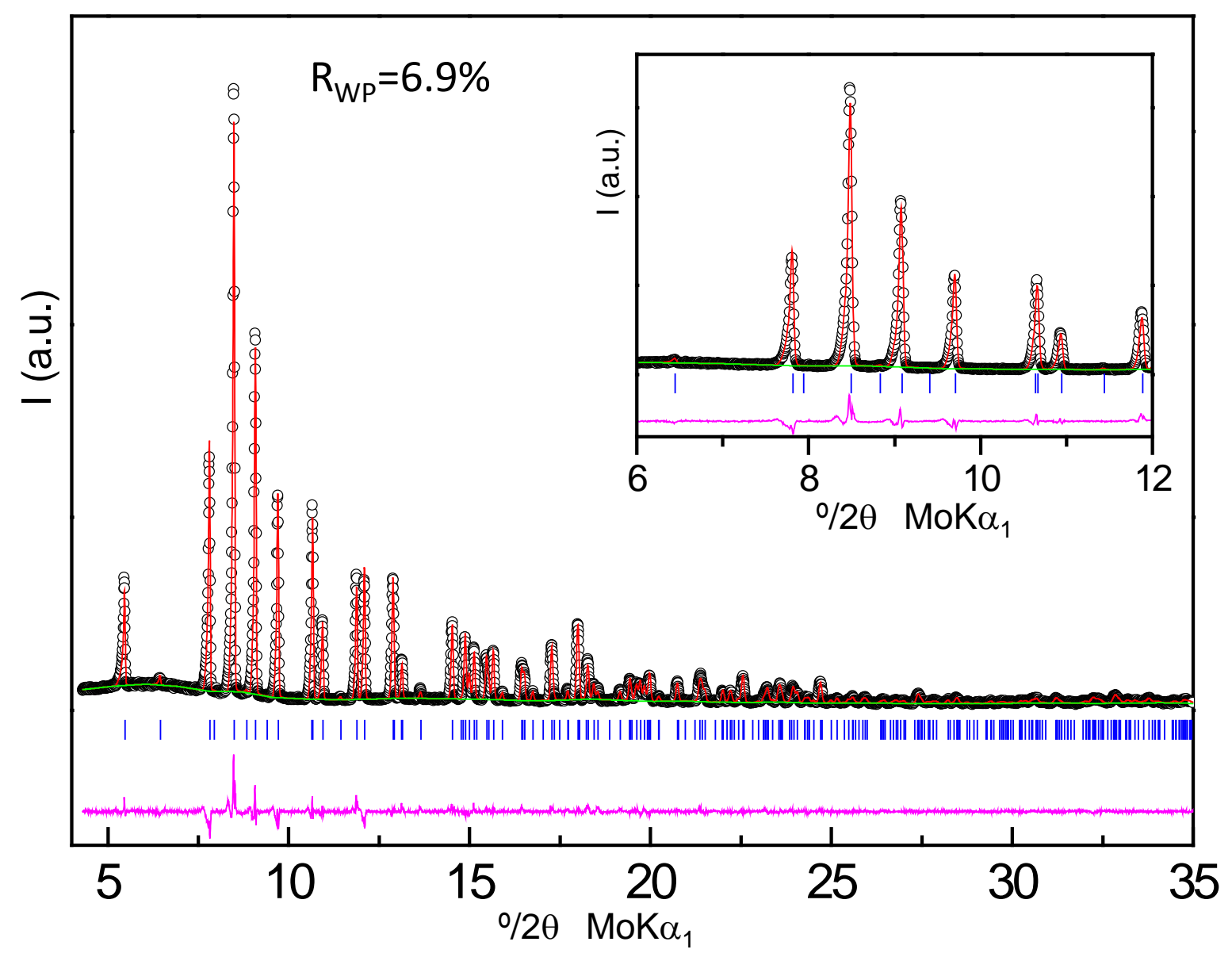

Figure S4. Mo-K $\alpha_{1}$ radiation Rietveld plot for xylose. 


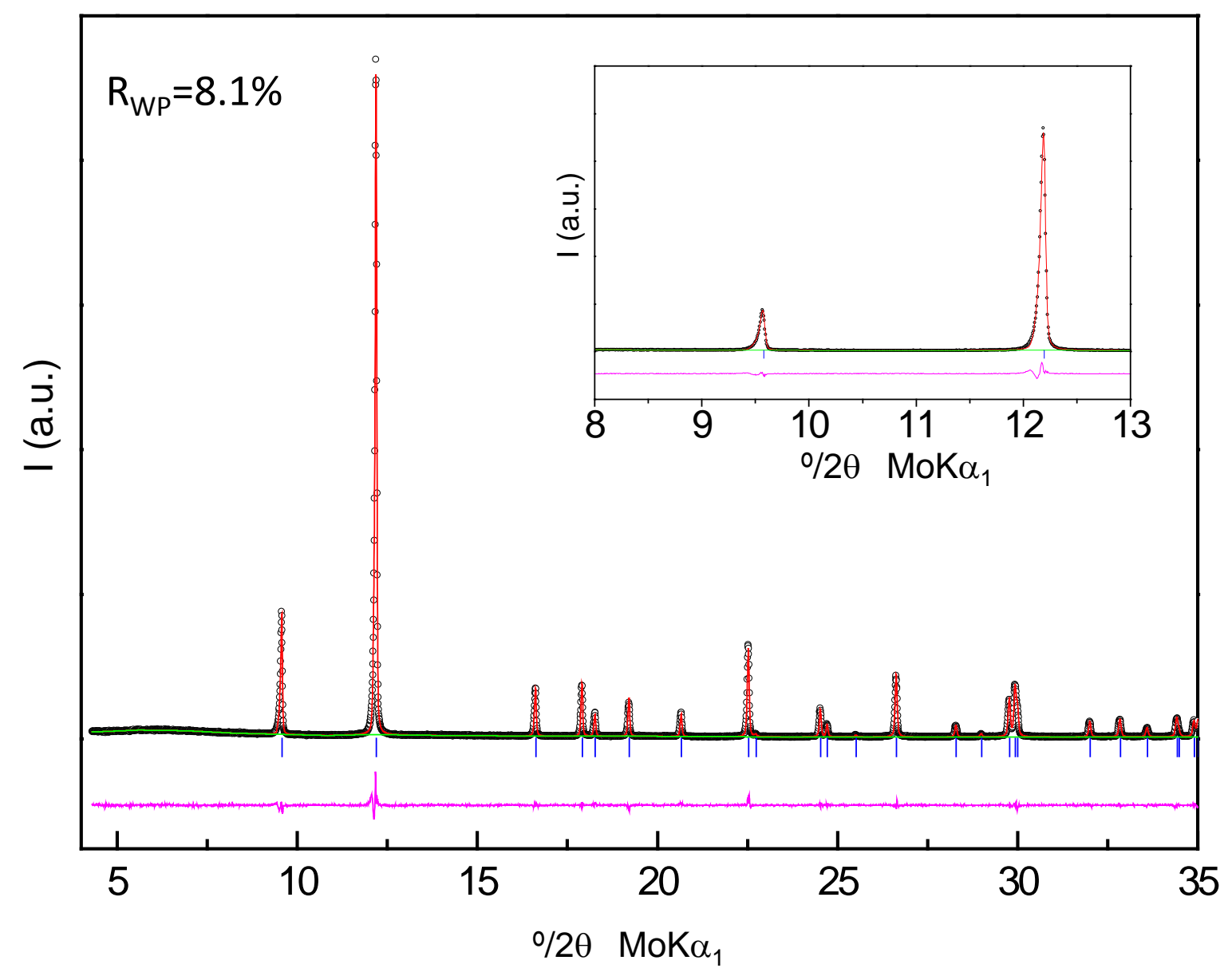

Figure S5. Mo-K $\alpha_{1}$ radiation Rietveld plot for quartz. 


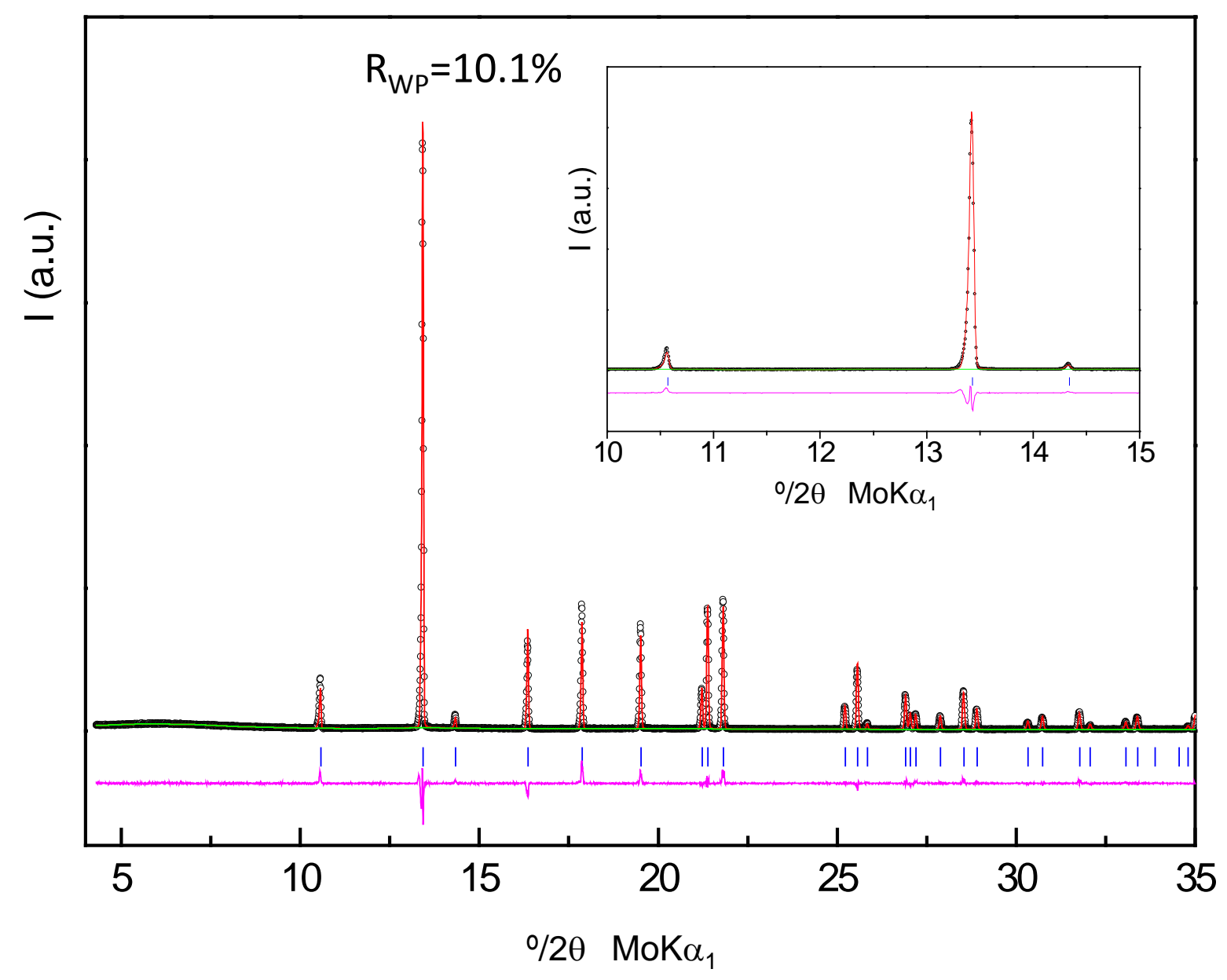

Figure S6. Mo-K $\alpha_{1}$ radiation Rietveld plot for calcite. 


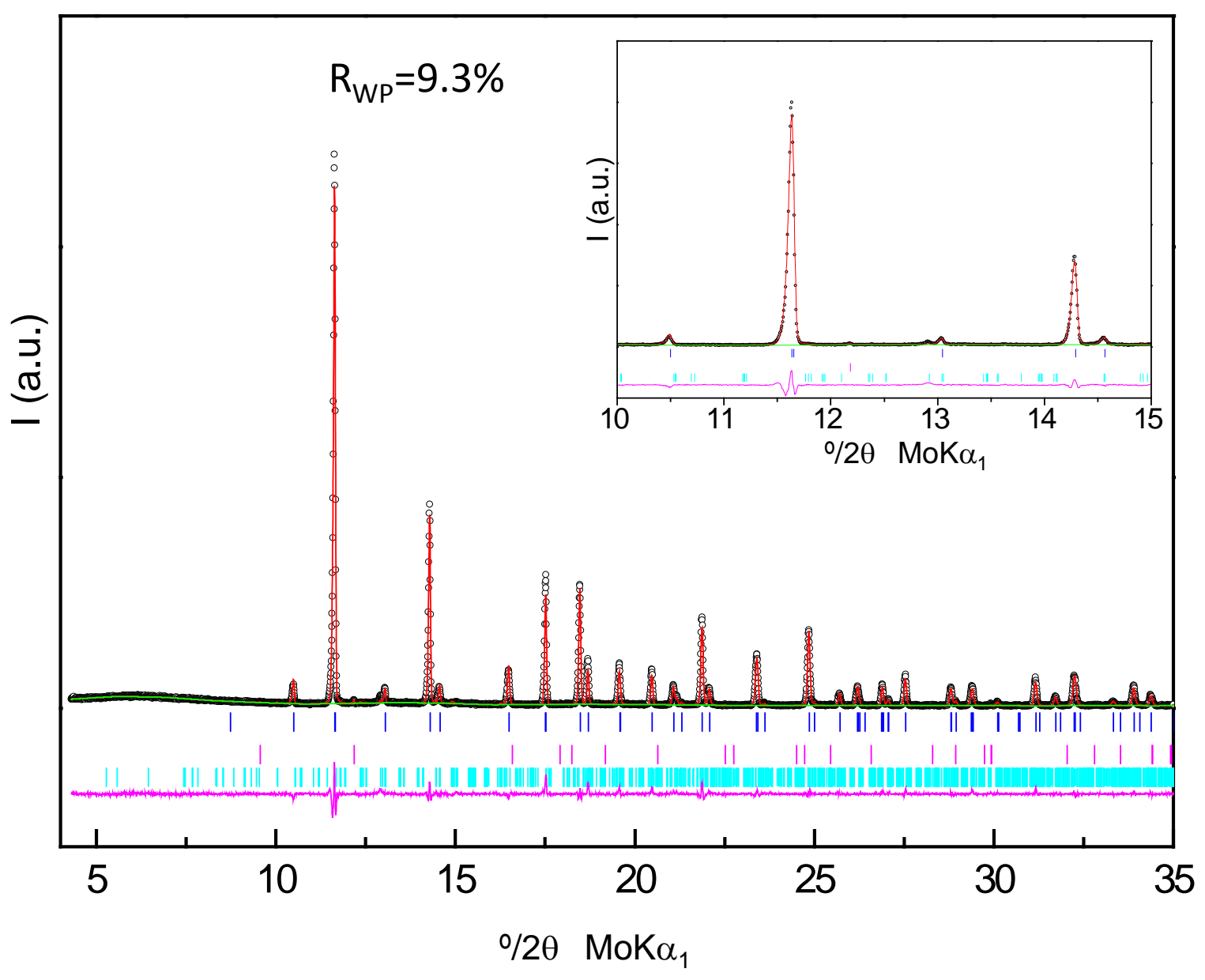

Figure S7. Mo-K $\alpha_{1}$ radiation Rietveld plot for insoluble anhydrite. 


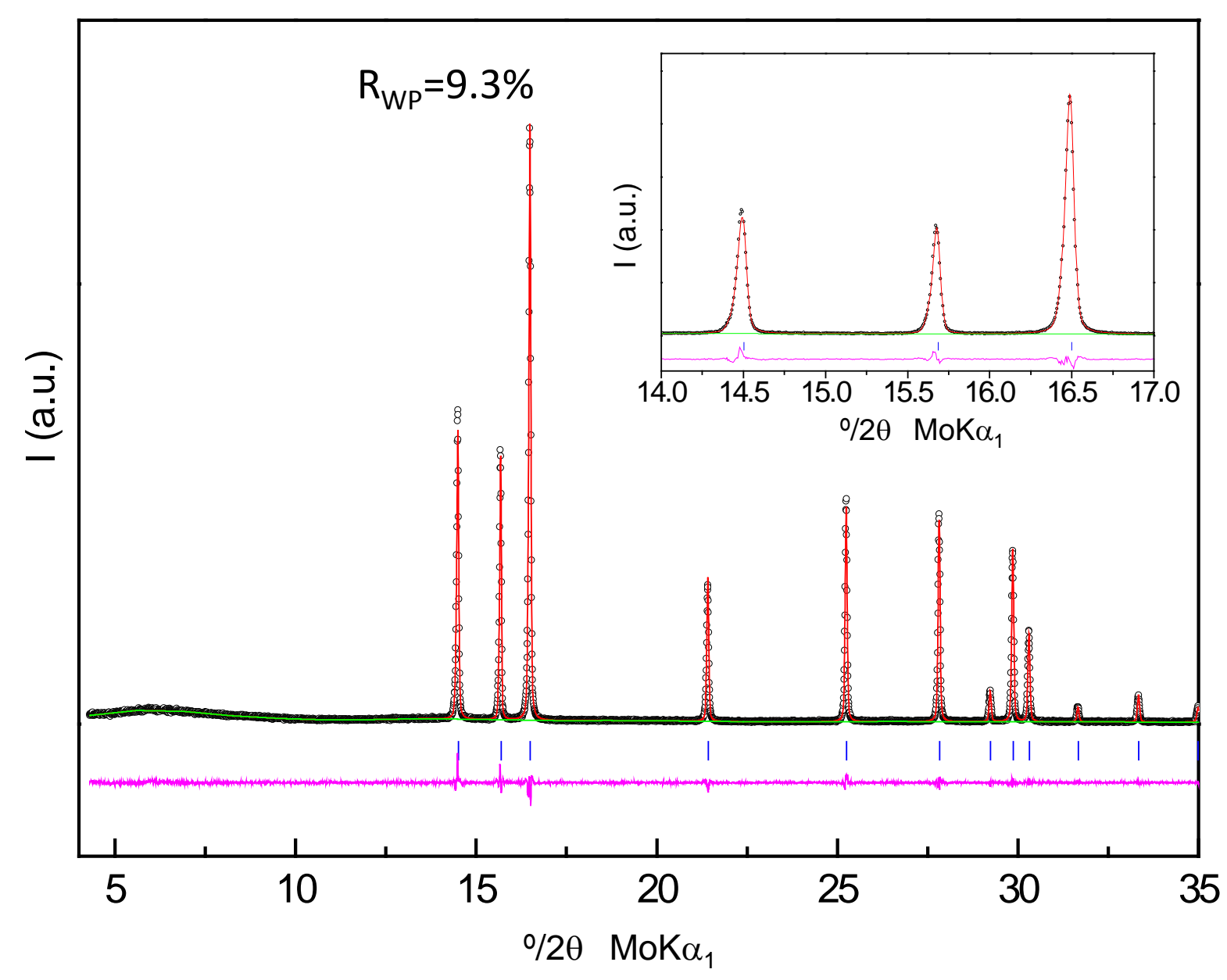

Figure S8. Mo-K $\alpha_{1}$ radiation Rietveld plot for zincite. 

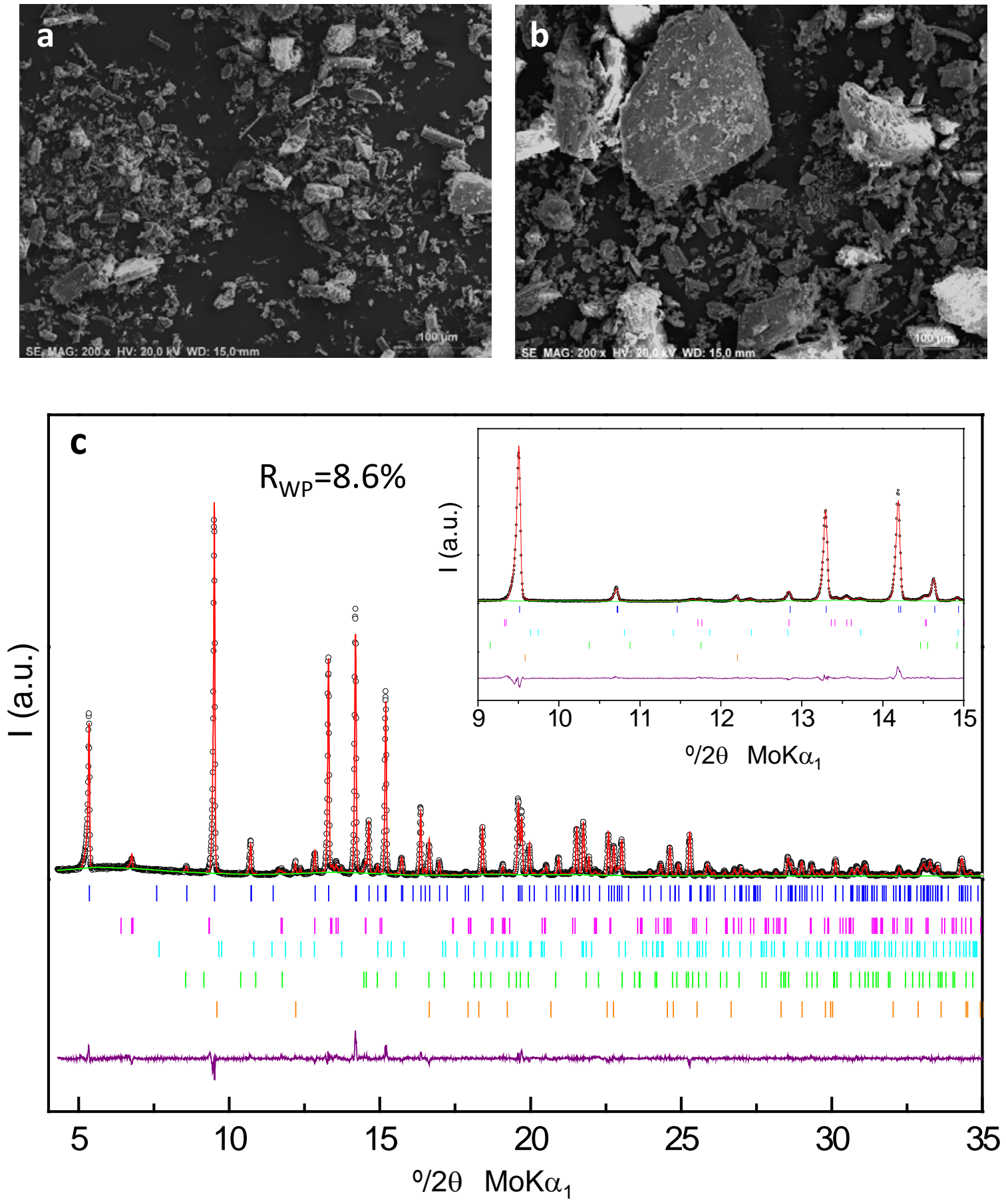

Figure S9. Scanning Electron Microscopy micrographs for (a) micronized gypsum, and (b) ground single crystal gypsum. (c) Mo-K $\alpha_{1}$ radiation Rietveld plot for micronized gypsum. 


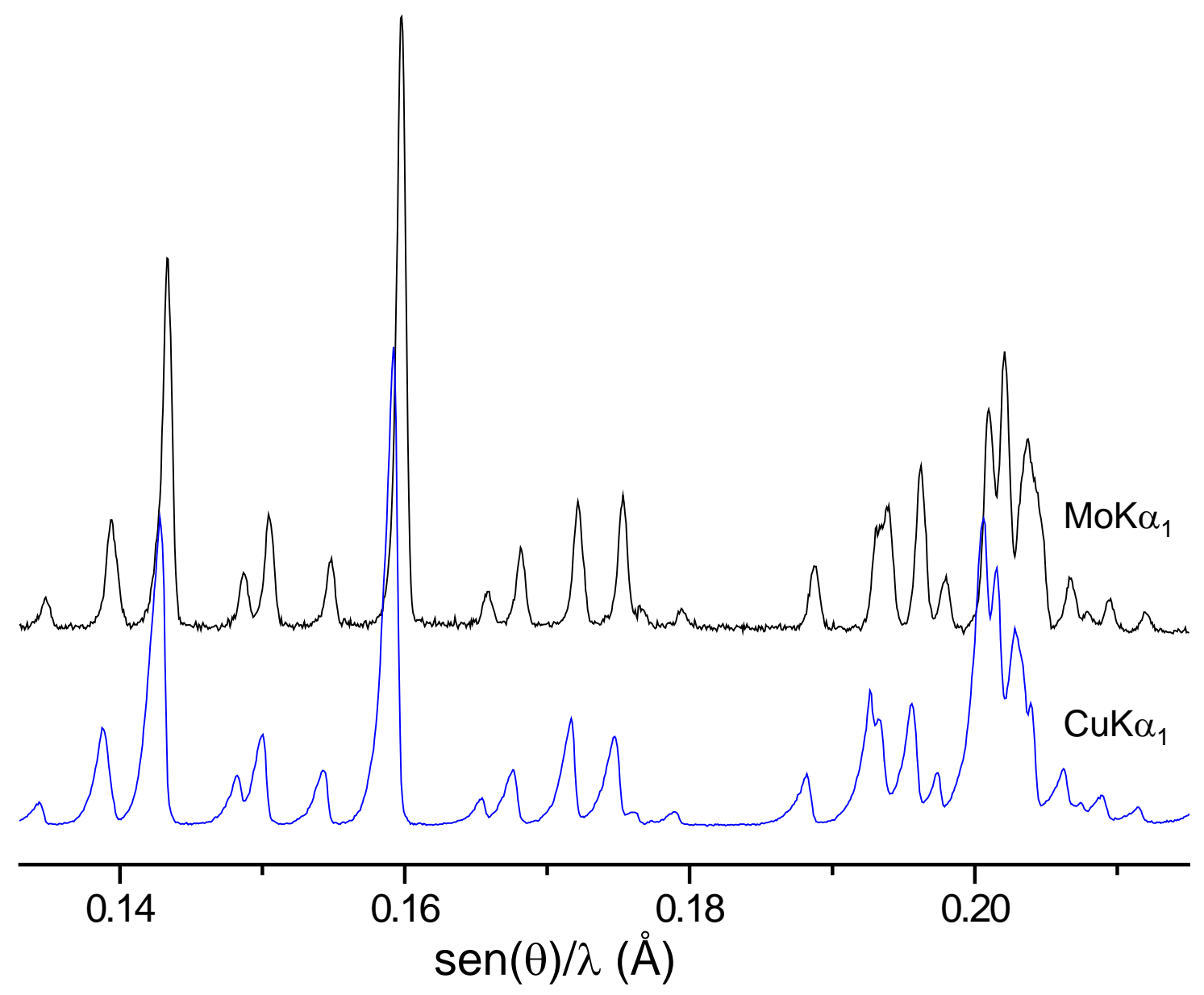

Figure S10. Mo-K $\alpha_{1}$ raw pattern (black) for glucose collected in transmission geometry. Cu-K $\alpha_{1}$ raw pattern (blue) for glucose collected in reflection geometry. 